464. Otto N. Witt und Otto Braun: Uber Umlagerungen in der Gruppe der Aceto-naphthole.

[Ans dem Techn.-chem. Institut der Technischen Hochschale za Berlin.] (Kingeg. am 30. November 1914; vorgetr. in der Sitz. von Hrn. Otto N. Witt.)

Im Jahre 1888 beobachtete der eine ron uns') bei dem Versuche, $\alpha$-Naphthol in Eisessiglösung zu sulfieren, das Auftreten eines ia Wasser unlöslichen Nebenproduktes von phenolartigem Charakter, welches als ein Oxynaphthyl-methyl-keton erkannt wurde und infolgedessen den Namen Aceto-naphthol erhielt. Es wurde festgestellt, daß durch Erbitzen von Naphtbol, Eisessig und Chlorzink die Ausbeute an Acetonaphthol auf $30 \%$ sich steigern lieB. Eine Rejhe von Derivaten des neuen Körpers wurde hergestellt, welche die große Reaktionsfähigkeit desselben dartat. Bezüglich der Konstitution des Acetonaphthols mußte angenommen werden, daß die Hydroxylgruppe ebenso wie im $\alpha$-Naphthol in der $\alpha$-Stellung sich befände, während für die neu eingetretene Acetylgruppe die Wabl zwischen der ortho- und der para-Stellung zum Hydroxyl freistand. Die Reaktionen des Acetonaphthols, insbesondere der Cbarakter der von ihm sich ableiteaden Azofarbstoffe ließen die Besetzung der ortho-Stellung als wahrscheinlich erscheinen, da aber ein Indophenol, wie ein Körper mit freier para-Stellung zur Hydroxylgruppe es hätte liefern sollen, damals nicht erbalten werden konnte, so wurde eine endgültige Entscheidung über die Stellung der Acetylgruppe im Molekül weiteren Untersuchungen vorbehalten.

Das Aceto-naphthol ist ein durch seine Eigenart 80 interessanter Körper, daß es seit der Zejt seiner Entdeckung im hiesiged Laboratorium immer wieder den Gegenstand von Untersuchungen gebildet hat, deren Resultate allerdings nicht veröffentlicht wurden. Das Acetonaphthol batte die Aufmerksamkeit auch anderer Chemiker auf sich gezogen, deren Beobachtungen die hier angestellten vielfach vorwegnahmen. Andere Untersuchungen mußten aus anderen Gründen unverñffentlicht bleiben.

So hatte sich im Laufe der Zeit ein reiches, aber wenig zusammenhängendes Material von Beobachtungen angehäuft, welches bei erneuten Arbeiten verwendet werden konnte, zugleich aber durch neue Versuche ergänzt werden mußte. Dis nachfolgende Untersuchung ist das Ergebnis einer derartigen Neubearbeitung gewisser aus dem Studium des Acetonaphthols sich ergebender Fragen.

J) Otto N. Witt, B. 21, 321 [1888]. 
Das Acetonaphthol war eigentlich nicht der erste bekannt gewordene Vertreter der von ibm repräsentierten Gruppe von Verbindungen. Schon 1886 hatte H. Erdman n auf der damaligen Naturforscherversammlung kurz über ein Kondensationsprodukt der Benzallävulinsäure berichtet, welches bei der Destillation dieser letzteren unter Wasserabspaltung sich bildet und nichts andres sein konnte als ein $\alpha$-Naphthol, in welchem die meta-Stellung zur Hydroxylgruppe durch den Acetylrest besetzt war. Dieser von seinem Entdecker als - Ketonaphtbolk bezeichnete Körper war also ein Isomeres des Acetonaphthols. In einer in diesen $\gg$ Berichten ${ }^{1}$ ) veröffentlichten Arbeit macbte Erdmann auf die naben Beziehungen 2 wischen beiden Substanzen aufmerksam und teilte mit, dab sein Produkt, im Gegensatz zum Acetonaphthol, mit großer Leichtigkeit die Indopbenolreaktion gebe.

Bald stellte sich ein drittes Isomeres ein, welches ebenfalls schon seiner Bildungsweise nach die Acetylgruppe in demselben Benzolring des Naphthalins enthalten mußte, in dem auch die Hydroxylgruppe sich befindet. Gattermann, Ehrhardt und Maisch wandten die Friedel-Craftssche Synthese auf Phenolätber an und erbielten dabei aus dem Methyl- und Äthyläther des $\alpha$-Naphthols durch die Einwirkung von Acetylchlorid und Chloraluminium zwei Ketone von den Schmelzpunkten $71-72^{\circ}$ und $78-79^{\circ}$, für welche sie, nach Analogie mit dem üblicben Verlauf der benutzten Reaktion, die para-Stellung der Acetyl- zur Methoxyl- bezw. Äthoxylgruppe annahmen "). Einige Jahre später veröffentlichten ${ }^{3}$ ) Hartmann und Gattermann die Beobachtung, daß derartige Äther durch Behandlung mit Aluminiumchlorid leicht verseift werden und dabei die freien Hydroxylabkömmlinge von Ketonen liefern. Aus dem obengenannten Äthyläther voln Schmp. $78-79^{\circ}$ konnte so das zugrundeliegende Aceto-naphthol rom Schmp. $98^{\circ}$ gewonnen werden, welches ebenso wie die beiden andren damals bekannten Körper dieser Zusammensetzung in schönen Nadeln krystallisierte.

Nachdem auf solche Weise drei Vertreter dieser Körperklasse bekannt geworden waren, welche alle die Acetylgruppe in demselben Benzolring des Naphthalins mit der Hydroxylgruppe enthalten mußten, konnte Friedländer ${ }^{4}$ ), der im Jahre 1895 obenfalls sich dem Gegenstande zuwandte, die von dem einen von uns seinerzeit offen gelassene Frage nach der relativen Stellung der Seitenketten im Acetonaphtbol durch den nabeliegenden Schluß beantworten, daß die ron dem Ent-
1) B. 81, 635 [1888].
2) B. 88,1208 [1890].
3) B. 25, 3534 [1892].
b) B. 28, 1946 [1895]. 
decker des Acetonaphthols für wahrscheinlich gehaltene ortho-Stellung derselben, zu einander die einzig mögliche sei, da ja die von Erdmann und $G a t t e r m a n n$ hergestellten Isomeren zweifellos die Inbaber der meta- und para-Stellungen repräsentierten.

Die drei Isomeren werden somit durch die nachfolgenden Konstitutionsformeln dargestellt:

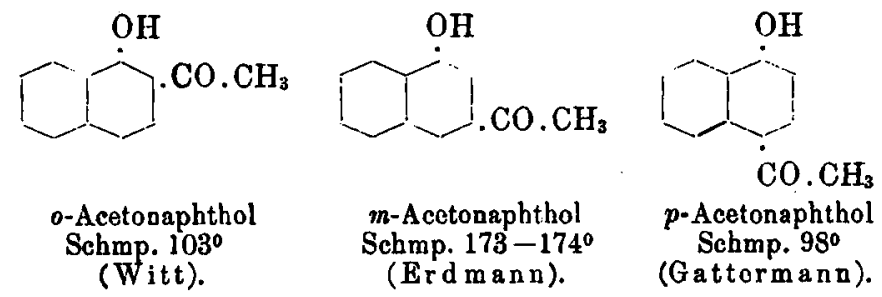

Für eine oberflächliche Betrachtung erscheint auf solche Weise die ganze Angelegenbeit als erledigt. Auch blieben weitere scbärfere Jeweise für dịe von dem einen von uns unsprünglich nur mit Vorbebalt angenommene ortho-Stellung der Seitenketten in dem ron ibm entdeckten Acetonaphtbol nicht aus. Das Versagen der Indophenolreaktion, welches das wichtigste Bedenken gegen die Annabme einer freien para-Stellung gebildet hatte, wurde als eine rein experimentelle Schwierigkeit erkannt, welche sich durch die Wabl eines geeigneten Uxydationsmittels beseitigen ließ. Versetzt man eine Lösung von $\gamma$-Phenylendiamin (freie Base) und Acetonaphthol in Alkohol mit einer, mit Ammoniak bis zur Wiederlösung des gefällten Oxyds versetzten J.ösung von Silbernitrat, so tritt langsam in der Kälte, rascber beim Erwärmen eine rein himmelblaue Färbung auf, wäbreod gleichzeitig netallisches Silber in weißen Krystallen, z. T. auch als spiegelnder Belag des Glases sich abscheidet. Aus der alkoholischen Löзung läßt sich der blaue Farbstoff gewinnen und schon durch seine große Empfindlichkeit gegen Säuren als typisches Indophenol erkènnen.

Für die ortho-Stellung der Seitenketten im Acetonaphthol spricht, wie schon Friedländer hervorgehoben bat, auch der Widerstand, welchen es seiuer Alkylierung eutgegensetzt. Nur bei sebr energischer Bebandlung und auch dann nur mit sehr schlechten Ausbeuten konnten der Äthyl- und Methyläther als dickflicssige Öle erhalten werden, während die von $G$ attermann bergestellten, oben erwähnten paraVerbindungen schön krystallisierende Substanzen sind.

Den stärksten Beweis für die ortho-Stellung der Seitenketten im Acetonaphthol bilden indessen die im Laule der Jahre mit ihm ausgeführten Synthesen, bei welchen die Seitenketten unter Zuhilfenabme weiterer Atomkumplexe zu Ringschließungen benutzt wurden. So 
stellte G. Ullmann') durch intramolekulare Kondensation des Acetylacetonaphtbols ein Naphtho-cumaran dar und St. v. Kostadecki ${ }^{2}$ ), sowie seine Schülerin Gertrud Woker ${ }^{3}$ ) führten die durch Anwendung $\operatorname{der}$ Claisen schen Reaktion auf das Acetonaphtbol erbältlichen $>\mathrm{Chal-}$ koner, deren erstes, vom o-Nitrn-benzaldebyd abgeleitetes Beispiel schon von dem einen von uns in dessen ursprünglicher Abbandlung über das Acetonaphthol erwähnt wurde, in Flavone, Flavanone und Flavonole über. Äbnliche Resultate waren schon früber bei einer im hiesigen Laboratorium ausgeführten und leider durch ein Ungliick verloren gegangenen Arbeit erhalten worden.

Die immerhin auffallende Tatsache, daß bei einem Substitutionsvorgang am $\alpha$-Naphthol die neu eintretende Gruppe nicht die freie para-, sondern die ortho-Stellung zum Hydroxyl aufsucht, wurde einigermaßen verständlich durch Versuche, welche der eine von uns zu dem $Z$ wecke anstellte, die Darstellungsweise des Acetonaphthols zu verbessern. Es zeigte sich dabei, daß das Acetonaphthol nicht das primäre Produkt der Einwirkung von Eisessig auf $\alpha$.Naphthol bei Gegenwart wasserentziehender Mittel ist, sondern durch intramolekulare Umlagerung aus zunächst gebildetem $\mathrm{Naphtbyl-acetat} \mathrm{entsteht.} \mathrm{Als}$ praktisches Resultat dieser Beobachtung ergab sich die Tatsache, daß bei der Heratellung ron Acetonaphthol durch Zusatz von Essigsäureanhydrid zu dem Gemisch der Ingredienzen die Ausbeute auf $80 \%$ und darüber von dem Gewjcht des angewandten Naphthols gesteigert werden kand.

Man Jöst zweckmäßig $30 \mathrm{~g}$ Cblorzink in $30 \mathrm{ccm}$ Eisessig, fügt $45 \mathrm{~g}$ Essigsäureanbydrid und $60 \mathrm{~g}$ Naphthol zu und bält das Gemisch $1 / 2$ Stunde lang bei $140^{\circ}$ in lebhaftem Sieden am Rückflußkühler. Man gießt dann in heißes Wasser und rübrt das sich ausscheidende schwere, dunkelrote Öl damit tüchtig durch, um das Chlorzink zu entfernen. Bei langsamem Erkalten erstarrt das Öl zu einem Krystallbrei, der mit kaltem Alkobul durchgeknetet und zur Entfernung des roten Farbstoffs ausgewaschen wird. Die zurückbleibenden hellgrauen Krystalle werden aus siedendem Alkohol umkrystallisiert und liefern dabei bis zu $55 \mathrm{~g}$ Acetonaphthol in apfelgrünen Krystallen rom Scbmp. $101-103^{\circ}$.

Wenn man nun diese Krystalle zum Zwecke der Erzielung eines Produktes von vollkommenster Reinheit mehrfach und aus verschiedenen Lösungsmitteln umkrystallisiert, so drängen sich dabei zwei eigentümliche Beobachtungen auf. Einerseits ist die Farbe des Produktes nie ganz gleich, sondern bald etwas beller, bald etwas dunkler, bald mehr grünlich, bald mehr strohgelb. Auch ändert sich die Farbe am
J) B. 30, 1466 [1897].
ๆ) B. 81, 705 [1898].
3) B. 39, 1649 [1906]. 
Licht, ohne daß dabei eine Zersetzung zu beobachten wäre. Andrerseits ändert sich der Schmelzpunkt des Präparates in ziemlich regelloser Weise und zeigt jedenfalls nicht bei zunehmender Reinigung das sonst meist beobachtete Ansteigen, sondern vielmehr ein Sinken. Präparate, welche im Anfang bei $101^{\circ}$ und sogar bei $102-103^{\circ}$ schmolzen, zeigen nach mehrfacher Rekrystallisation meist den Schmp. 98-99 ${ }^{\circ}$, d. h. genau dieselbe Verflüssigungstemperatur, welche auch Gattermann für sein $p$-Acetonaphthol gefunden hat.

Das Problem der Farbe des Acetonaphthols ist schon in der mebrfach erwäbnten ersten Arbeit des einen von uns berührt worden, indem er darauf hinwies, daß das Acetonaphthol in Sinne seiner Theorie über den Zusammenhang zwischen Konstitution uud Farbstoffnatur organischer Verbindungen als ein Farbstolf aufzufassen sei, als ein primitiver Vertreter der Familie der Oxy-keton-Farbstoffe, zu welcher als vollendetste Repräsentanten ja aucla die Alizariofarbstoffe gebören. Wie diese, enthält das Acetonaphthol, obgleich es noch nicbt die Merkmale eines technisch verwendbaren Farbstoffes aufweist, die Ketogruppe - $\mathrm{CO}$ - als chromophore und Hydroxyl als auxochrome Gruppe. H. Erdmann ging in seiner angezogenen Abhandlung ebenfalls auf diese Frage ein. Er wies darauf bin, daß sein $m$-Acetonaphthol im freien Zustande weiß und nur in der Form seiner Salze gelb sei und glaubte voraussagen zu dürfen, daB das jetzt mit Sicherbeit als ortho-Verbindung erkannte Acetonaphthol des einen von uns, für welches er aber die para-Stellung der Seitenketten für wabrscheinlicher hielt, sich sbei genügender Reinigung« im freien Zustande ebenfalls als weiß, in seinen Salzen aber als gelb erweisen würde.

In dieser Annabme bat Erdmann sich getäuscbt, das Acetonaphthol blieb trotz der oben erwähnten Farbenschwankungen doch immer gelb. Es verhält sich damit ähnlich wie mit den Nitrophenolen und den Nitroso- $\alpha$-naphtbolen, von welchen auch die ortho-Isomeren gelb, die andren aber weiß sind Von dem zur Zeit des Erscheinens der Abhandlung von Erdmann noch unbekannten $p$-Acetonaphthol mußte man in der Tat erwarten, daß es weiß sein würde. Gattermanns Mitteilung, da $B$ auch die von ibm gewonnene $p$-Verbindung gelb sei, bedeutete rom farbentechnischen Standpunkte eine Utberraschung.

Die am o-Acetonaphthol beobachteten Schwankungen in der Färbung und wohl auch im Schmelzpunkt lassen sich erklären, wean man mit $\mathrm{Hantzsch}^{1}$ ) nicht nur annimmt, daß dieses Oxyketon in zwei tautomeren Formen existieren kann, von denen die eine gelb, die andre aber farblos ist und $\mathrm{daB}$ diese verschiedenen Formen in den

1) B. 39, 3096 [1906]. 
verschiedenen Abkömmlingen des Oxyketons zur Geltung kommen, oondern dả es auch im freien Zustande bald die eine, bald die andre Gestalt annehmen kann und daB die durch Krystallisation aus irgend welchen Medien erbaltenen Präparate starre Lösungen der farbigen in der farblosen Modifikation sind, welche natürlich je nach dem Vorwalten der einen oder der andren eine verschiedene Intensität und Nuance ibrer gelbeu Färbung zeigen werden. Gerade die von Ha b z s ch mitgeteilte Beobachtung, daß eine Lösung des o-Acetonapbthols in Ligroin im Gegensatz zu allen andren Lösungen dieses Körpers farblos ist, ist ein Beweis dafür, daß bei diesem Oxyketon die farblose Modifikation nicht nur in ihren Acyl- und Alkyl-Abkömmlingen, sondern auch in Lösung vorkommen kann, - weshalb sollte sie nicht in der farbigen Modifikation gelöst existieren können? Ebenso könnte aucb die Veränderung der Farbe des Acetonaphthols am Licht, die als ein teilweises Ausblassen bezeichnet werden $\mathrm{kann}$, auf einer $\mathrm{Zu}$ nahme seines Gebaltes an farbloser Nodifikation beruhen.

Auf solche Weise erklären sich die beobachteten Anomalien in der Färbung und auch im Schmelzpunkt des o-Acetonaphthols, nicht aber die auffallende Übereinstimmung seines Schmelzpunktes mit dem des von Gattermann hergestellten angeblichen para-Derivates. Hier mußte zunächst eine genaue Vergleichung beider Substanzen vorgenommen werden. Wir baben daber das Gattermannsche Produkt nach den Angaben seines Entdeckers bergestelit und zu unserer Überraschung gefunden, daß es mit dem 0 -A cetonaphthol zweifellos identisch ist. Es zeigte sich volle UUbereinstimmung in Farbe, Krystallform und -babitus und ein Gemisch beider Substanzen schmolz genau bei derselben Temperatur, wie jeder der Gemengteile für sicb, nämlich etwas unscharf bei $98-99^{\circ}$. Hieraus ergab sich, daß das $G$ attermannsche Produkt nicht die nun immer noch fehlende para-Verbindung, sondern das ortho-Derivat war. Ein scharfer Beweis für die Konstitution gerade dieses Produkts war ja auch von seinem Entdecker nicht gegeben worden, er hatte vielmehr die para.Stellung der Substituenten nur deshalb für gegeben erachtet, weil sie bei der Einfübrung ron Substituenten durch die Friedel-Craftssche Reaktion in der Regel aufgesucht zu werden pflegt, doch war eine gelegentliche Ausnahme immerhin nicht ausgeschlossen.

Als Schwierigkeit blieb immer noch die Tatsache bestehen, daß die von Gattermann zunächst gewonnenen Äthyl- und Methyläther seines Acetonaphthols schön krystallisierende und hochschmelzende Substanzen warea, während Friedländer durch die Äthylierung und Methylierung des o-Acetonaphthols Óle erbalten hatte, welche auch bei niedriger Temperatur flüssig blieben. 
Diesen Widerspruch baben wir aufgeklärt durch ein genaueres Studium der Gattermannschen Reaktion. Diese verläuft keineswegs glatt, sondern sehr stürmisch unter gleichzeitiger Entstebung größerer Mengen von teerigen Nebenprodukten. Diese hatte Gattermann als zum größten Teil in Alkali löslich erkannt und aus diesem Belund die Anregung dazu entnommen, die erbaltenen Äther durch Behandlung mit Aluminiumchlorid zu verseifen.

Bei der Durchführung der Gattermannschen Synthese wird Schwefelkohlenstoff als Lösungsmittel verwandt. In $500 \mathrm{ccm}$ davon werden $86 \mathrm{~g} \alpha-\mathrm{Naphthol-äthyläther} \mathrm{und} 60 \mathrm{~g}$ Acetylchlorid gelöst, das auf dem Wasserbade siedende Gemisch wird portionsweise mit im ganzen $100 \mathrm{~g}$ Aluminiumchlorid versetzt. Die anfangs stürmische Salzsäure-Entwicklung kommt nach etwa $2^{1 / 2}$ Stunden zum Stillstand, es hat sich dann eine Alumioiumchlorid-Verbindung des Reaktionsprodukts als dicker, grünschwarzer Teer am Boden des Gefäßes ausgeschieden, wäbrend der überstebende Schwefelkoblenstoff nichts wesentliches mehr enthält. Nach dessen Abgießen wird der Teer mit Wasser zersetzt, der nun noch vorhandene Schwefelkohlenstoff wird mit Dampf abgeblasen. Es hinterbleibt ein schwarzer harziger Kuchen, aus welchem $G$ attermann seinen Acetonaphtbol-ätbyläther durch Herauslösen der Verunreinigungen mit Natronlauge und Rekrystallisation des verbleibenden Rückstandes erbalten hatte.

Wir baben, gerade um das Wesen der eingetretenen Reaktion etwas genauer kennen zu lernen, die beschriebene Aufarbeitungsmethode etwas modifiziert. Der oben erwähnte harzige Kuchen wurde zunächst in Äther aufgenommen, wobei eine geringe Menge eines kobligen Pulvers zurückblieb. Die ätherische Lösung wurde erschöpfeod mit Sodalösung ausgeschiitfelt. Aus den vereinigten gelbbraunen Sodalösungen wurde durch Salzsäure ein gelbes krystalliniscbes Produkt gefällt, welcbes durch metbodische $\mathrm{K}_{r y s t a l l i s a t i o n}$ aus Alkohol in zwei vollkummen farblose Verbindungen zerlegt werden konnte, eine schwerer lösliche vom Schmp. $140^{\circ}$ und eine aus den Mutterlaugen anschießende bei $198^{\circ}$ schmelzende.

Diese letztere ergab bei der Analyse die Zusammensetzung eines, Acetonaphtbols. In ihr liegt, wie weiterhin bewiesen werden konute, das zu dem Äthylăther vom Schmp. $78-79^{\circ}$ gehörende wirkliche $p$ Acetonapthnol vor, welches nun auch tatsächlich den böheren Scbmelzpunkt und die Farblosigkeit aufweist, wie man sie von der parct Verbindong dieser Reibe erwarten mußte.

Die Substanz rom Schmp. $140^{\circ}$ erwies sich als ein Diaceto$n$ aphthol, bei welchem also der Acetylrest $z$ weimal in das $\alpha$-Naphthol eingetreten war und zwar, wie weiterbin auch bewiesen werden 
konnte, in die ortho- und die para-Stellung. Es entspricht bezüglich seiner Stellung dem Martiusgelb oder 1.24. Dinitro-naphthol.

Die mit Soda erschöplte ätherische Lösung wurde mit verdünnter Natronlange ausgeschüttelt. Dabei eryab sich als einziges, durch teerige Verunreinigungen zunächst etwas maskiertes Produkt 4 Naphthol.

Der nun verbleibende reingelbe ätherische Auszug lieferte nach dem Abdestillieren des Äthers den Gattermannschen Acetonaphthol-ätbyläther rom Schmp. 78- $79^{\circ}$ in einer Ausbeute ron $56 \mathrm{~g}=52 \%$ der Theorie.

Dieser Ätber gebört nicht, wie bisher irrtümlich angenommen worden ist, zu dem schließlich aus ihm von Gattermann erbaltenen Acetonaphthol vom Schmp. $98^{\circ}$, sondern er ist der Äther des jetzt erst von uns aufgefundenen wirklichen $p$-Aceto-naphtbols rom Schmp. $198^{\circ}$. Bei der ohne besondere Vorsicbtsmaßregeln durchgefübrten Verseifung dieses Äthers mit Hilfe ron Aluminiumcblorid, wie Gattermann sie gewäblt batte, findet eine Umlagerung statt, die Acetylgruppe wird aus der para- in die ortho-Stellung transportiert. Die gleichzeitig ab. und wieder aufbauende Tätigkeit des Aluminiumchlorids geht noch viel weiter, sie führt in der einen Richtung zunächst zur Rückbildung ron $\alpha$-Naphthol, in der andren zur Einführung abgespaltener Acetylgruppen in die Moleküle des $\alpha$-Naphthols und des $o$ - und $p$-Acetonaphthols und somit zur Bildung des o-Acetonaphtbols und des oben erwähnten Diaceto-naphthols rom Schmp. $140^{\circ}$.

Die Richtigkeit dieser Erkenntnis konnten wir beweisen, als es uns gelang, die allzu stiirmische Reaktion des Aluminiumchlorids durch Wahl eines andren Verdünnungsmittels zu mäßigen und in die Hand zu bekommen. Ein solches Mittel wurde in reinem Benzol gefunden, welches früher schon von F. Ullmana und Brittuer ${ }^{1}$ ) in einem ähnlichen Falle benutzt worden war. Bei Verwendung dieses Lösungsmittels konnten die synthetischen sowobl wie die spaltenden Vorgänge pinigermaßen geleitet und veriolgt werden. Die Aufarbeitungen geschaben stets durch das beschriebene Verfabren der folgeweisen Extraktion mit Sodalösung und verdännter Natronlauge und Zurückbaltung der Neutralkörper in der ätherischen Lösung. Dabei nimmt Sodalösung das stark saure $p$ - und Diaceto-naphthol auf, Natronlauge das 0 -Acetonaphthol und a-Naphthol und als neutral erweisen sich die Äther dieser Substanzen.

1) B. 42,2545 [1909]. 
Durch die auf solche Weise verbesserte Metbode der Aluminiumchlorid-Behandlung konnte der Gattermannscbe Äther vom Schmp. $78-79^{\circ}$ mit einer Ausbeute von etwa $80 \%$ der Theorie in das von uns aufgefundene Acetonaphthol vom Schmp. $198^{\circ}$ übergeführt und damit der Beweis erbracht werden, $d a B$ beide zusammengehören. Kaliumpermanganat oxydiert das neue Acetonaphtbol zu Phtbalsäure, womit bewiesen ist, $d \mathfrak{d} \beta$ beide Seitenketten sich in demselben Benzolring des Naphthalins befinden. Hier wird nuv die SchluBfolgerung, daß bei deun Bekanntsein der ortho- und meta-Verbindung für das neue Isomere vur die para-Stellung übrig bleibt, unbedingt zulässig. Außerdem erinnern die aus ihm berstellbaren Azofarbstoffe in ibrer Unempfindlichkeit gegen Säuren, ibrer scharlachroten Farbe und ihrem ganzen Charakter an die so sehr eigenartigen ortho-Azofarbstoffe des $\alpha$-Naphthols.

Das Diaceto-naphtbol bildet stets das Nebenprodukt des $\boldsymbol{p}$-Acetonaphthols bei der Verseifung des Äthers vom Schmp. $78-73^{\circ}$ durch Aluminiumcblorid, es muB also aus dem $p$-Acetonaphthol entstehen. Wie dieses wird es durch Permanganat zu Phthalsäure oxydiert, entbält also aucb alle Seitenketten im gleichen Ring. Mit Diazo-Verbindungen reagiert es nicht mehr, die beiden Acetylgruppen müssen somit die ortho und para-Stellung zur Hydroxylgruppe besetzt halten, so daß sich ungezwungen die Annahme der Konstitution 1.2.4 ergibt. Durch Einwirkung von Hydroxylamin konnte sowobl ein Mono- als auch ein Dioxin erhalten werden.

Die im Vorstehenden kurz zusammengestellten Resultate unserer Untersuchung luden dazu ein, auch die vom $\beta-\mathrm{Naphthol}$ sich ableitenden Acetonaphthole darzustellen und etwas năher zu untersuchen. Der eine von uns batte schon bei der ersten Untersuchung des $o$ Aceto- $\alpha$-naphthols sein neues Verfabren auch auf das $\beta$-Naphthol angewaudt, dabei aber nur in Alkali unlösliche Produkte erbalten, welche nicht weiter untersucht wurden. Später hat Gattermann seine Aluminiumchlorid-Methode auch auf den $\beta$-Naphthol-methyl- und -äthyläther angewandt und dabei zwei bei $57-58^{\circ}$ und $62-63^{\circ} \mathrm{schmel-}$ zende Acetonaphthol-äther erbalten, welche sich natürlich ron einem und demselben Aceto- $\beta$-naphthol ableiten müssen. Rousset ${ }^{1}$ ) stellte Betrachtungen über die Konstitution dieser Körper an und kam zu dem Schlusse, daß die Acetylgruppe in die $\alpha$-Stellung neben dem Hydroxyl eintreten müßte. Weiteres war über die Aceto- $\beta$-naphthole nicht bekanot geworden, als wir uns dem Gegenstand zuwandten.

ग) Bl. [3] 15, 633. 
Für unsere Versuche baben wir den im Handel unter dem Namen "Nerolin zugänglichen $\beta$-Naphthol-methylăther benutzt. Seine Kondensation mit Acetylchlorid wurde in der bereits beschriebenen Weise mit Aluminiumcblorid in Benzollösung vorgenommen und auch die Verarbeitung der Reaktionsmasse erfolgte in der gleichen $\Delta r$, wie es für die Derivate des $\boldsymbol{\alpha}$-Naphthols geschildert wurde. Die SodaAusschüttlung der ätherischen Rohlösung lieferte ein Acetonarb-

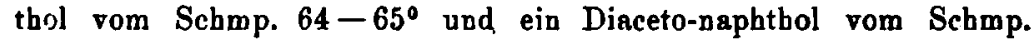
$165^{\circ}$. Das Natronlaugenextrakt enthielt neben harzigen Verunreinigungen zurückgebildetes $\beta-\mathrm{Naphthol.} \mathrm{Aus} \mathrm{der} \mathrm{ätberischen} \mathrm{Lösung}$ wurde der bereits von Gattermann beschriebene Aceto- $\beta$ - $n$ aphthol-methylätber vom Schmp. 57.5-58 ${ }^{\circ}$ gewunnen. Dieser lieferte bei der Aluminiumchlorid-Verseifung das eben erwähnte Aceto-naphthol rom Schmp. $64-65^{\circ}$ in einer Ausbeute von $78^{\circ}$ der Theorie.

Dieses Acetonaphthol wird ron Permanganat ebenso wie seine vom $\alpha$-Napbthol abstammenden Isomeren $2 u \mathrm{Phtbalsăure} \mathrm{oxydiert,}$ die Acetylgruppe muBte also in denselben Ring eingegriffen haben, in dem auch die Hydroxylgruppe sich befand. Aber die Scblußfolgerung von Rousset, daB es in die $\alpha$-Stellung neben dem Hydroxyl eingreifen müßte, erwies sich als unrichtig, denn das neue Acetonaphthol liefert mit Diazonium-Verbindungen leicht und glatt Azofarbstoffe. Solche entstehen bekanntlich aus Abkömmlingen des $\beta$-Naphthols nur dann, wenn die eben erwähnte $\alpha$-Stellung noch frei ist. Für den Eingriff dex Acetylgruppe blieben solchergestalt nur noch die 3- und 4-Stellung des $\beta$-Naphthols übrig. DaB in Wirklichkeit die erstere gewäblt und somit wiederum eiv o-A ceto-n aphthol gebildet wird, ergab sich aus der Fähigkeit des neuen Produkts, Kondensationen unter Ringschließung einzugehen. Durch Einwirkung von Hydrazin konnte nicht, wie ursprünglich beabsichtigt, ein zu weiterer Kondensation unter Wasserabspaltung befähigtes Hydrazon erbalten werden, sondern es vollzog sich sofort quantitativ die Bildung eines

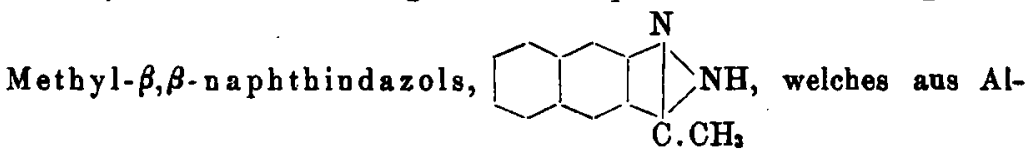
kohol in kleinen farblosen Nädelchen vom Schmp. $213^{\circ}$ krystallisiert und, wie von einem derartigen Körper zu erwarten war, sowobl in starker Natronlauge, als auch in starker Salzsäure sich löst.

Es sei hier beiläufig bemerkt, $d a B$ das alte o-Aceto- $a$-naphthol ein derartiges Verbalten nicht zeigt.

Das Diaceto. $\beta$-naphthol vom Schmp. $165^{\circ}$ liefert mit DiazoniumVerbindungen ebenfalls Azofarbstoffe. Durch Permanganat wird es 
zu einer bei $216-217^{\circ}$ schmelzenden Säure oxydiert, welche ein Auhydrid rom Schmp. 159-160" lieferte und sich somit als Trimellitsäure, HOOC: $\mathrm{COOH}$ . $\mathrm{COOH}$, erwies. Hier war also die zweite Acetylgruppe in den zweiten Benzolring des Naphtbalins und zwar in eine $F$-Stellung eingetreten. $\mathrm{Ob}$ dies nun eine 6-oder die 7-Stellung (Hydroxyl in 2) war, ließ sich nicbt entscheideu, docb rerdient, wenu man eine gewisse Analogie mit den Vorgängen bei der Sulfierung des $\beta$-Naphthols gelten lassen will, die Annabme der 6-Stellúng für den $\mathrm{zweiten}$ eintretenden Acetylrest den Vorzug.

Bei der Behandlang mit Hydrazin liefert das Diaceto- $\beta$-naphtbol je nach den angewandten Mengenverbältnissen zwei verschiedene Produkte, von denen das eine in haarfeinen, farblosen Nädelchen vom Schmp. $175^{\circ}$ krystallisiert und die Zusammensetzung eines A cetomethyl-naphthindazols besitzt, das andre; bei der Verwendung überschüssigen Hydrazins entstehende, ein citronengelbes, unschmelzbares, in allen Lösungsmitteln unlösliches Pulver bildet, in welchbem der Analyse nach auf zwei Reste des Diaceto-naphthols drei Hydrazinreste entbalten sind. Es ist also ein durch die Yerkettung zweier Moleküle des eben erwähnten Aceto-indazols durch ein Molekül Hydrazin entstandeues Hydrazon:

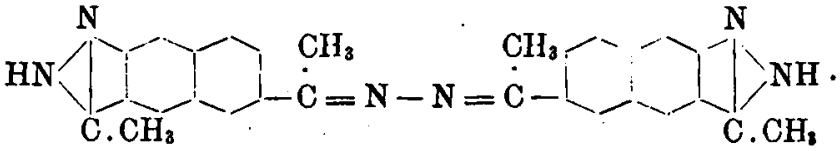

Durch Kochen mit starker Salzsäure wird Aceto-indazol vom Schmp. $175^{\circ}$ zurückgebildet.

Scbließlich ist nocb der Versuch gemacht worden, die Natur derjenigen Substanz aufzuklâren, welche der eine von uns im Jabre 1888 durch Bebandlung von $\beta$-Naphtbol mit Chlorzink und Essigsïure erhalten und als alkali unlöslich nicht weiter untersucht batte. Sie dürfte der Hauptmenge nach $\beta-N a p b t b y l a c e t a t$ gewesen seiv. Als wir die weiter oben beschriebene verbesserte Darstellungsmethode des Acetonaphthols unter Zusatz von Essigsäureanbydrid auf das $\beta$-Naphthol anwandien, wurde der Hauptmenge nach ein unerquickliches Harz erhalten. Nun wurde reines Naphtbylacetat der gleichen Be. bandlung unterworfen und dabei wieder ein harziges Produkt erbalten, aus dem sich aber in eiper Menge von wevigen Prozenten des angewandten Acetats ein Körper vom Schmp. $171^{\circ}$ und von der Zusammensetzung eines Acetonaphthols isolieren ließ. Er lieferte Azofarbstoffe, wurde durch Hydrazin in ein in gelben Nädelchen von Submp. $295^{\circ}$ krystallisierendes Hydrazon verwandelt und durch Permanganat 
7.u. Trimellitsäure abgebaut. Es lag hier also dasjenige Acetonaphthol des $\beta$-Naphthols vor, bei welchem die Acetylgruppe in den $z$ weiten Benzolring des Naphtbols und $z$ war in eine $\beta$-Stellung einfetreten war. Welche der beiden vorhandenen Stellungen dieser Art besetzt worden war, konnte natürlich wieder nicht entschieden werden, doch wird man auch hier wieder geneigt sein, nn eine Analogie mit der sog. Schaefferschen $\beta$-Naphthol-monosulfosäure zu denken und somit der Acetylgruppe die 6-Stellung zuzuweisen.

$\mathrm{Da} B$ bei dem Chlorzink-Veriahren ein ganz andres Produkt erbalten wird als bei der Aluminiumchlorid-Synthese, dürite hauptzächlich wobl den ganz verschiedenen Temperaturen zuzuschreiben sein, bei welchen beide sich vollziehen, denn während bei letzterer der Siedepunkt des Benzols eingehalten wird, muß das Gemisch aus $\beta$-Naphthylacetat, Chlorzink und Eisessig auf $150-160^{\circ}$ erhitzt werden, wenn es das Acetonaphthol vom Schmp. $171^{\circ}$ liefern soll.

$$
\begin{aligned}
& \text { Experimentelles. } \\
& \text { o-Aceto- } \alpha \text { - daphthol. }
\end{aligned}
$$

Nach der weiter oben gegebenen Vorschrift sind größere Mengen dieses Körpers dargestellt worden. Für seine Reinherstellung hat sich mehrfach wiederbolte Krystallisation aus Alkobol am besten bewäbrt. Bei langsamer Abküblung größerer Mengen der alkoholischen Lösung werden sebr schöne Krystalle erbalten. Das früher vorgeschlagene Verfabren der Ausfällung alkalischer Lösungen mit Kochsalzlösung hat sich als bedenklich erwiesen, da dabei mitunter eine plötzliche Zersetzung des ganzen Materials eintritt.

$$
p \text {-Aceto- } \alpha \text {-naphthol-äthyl-und-methyl-äther. }
$$

Die Herstellung dieser Körper geschab nach der Vorscbrift ron Gattermann in eivem Apparat, welcher mit Rückflußkübler, einem mit Quecksilber abgedichteten Rührwerk und einer Vorrichtung ausgestattet war, welche erlaubte, das vorber eingefüllte Aluminiumchlorid in kleinen Mengen zu der Flüssigkeit fallen zu lassed. Durch ein Wasserbad wird bis zum Sieden des Schwefelkohlenstoffs erwärmt. Die Reaktion kann dann durch Zugabe von Aluminiumchlorid eingeleitet und durch weitere Zugaben desselben im Gange erhalten werden. Die Mengenverbältnisse der Ingredienzien und dje Art der Aufarbeitung des Reaktionsgemisches sind bereits erwäbnt worden.

In genau derselben Weise wie in den Äthyl- kann die Acetylgruppe in den $\alpha$.Naphthol-methyläther eiugeführt werden, welcher sich dem Aluminiumchlorid gegenüber als weniger empfindlich erweist und infolgedessen nur sebr geringe Mengen der soda- und laugen-löslichen 
Zersetzungsprodukte, dafür aber bis zu $87 \%$ der Theorie an Acetonaphthol-methyläther liefert.

\section{$p$-Aceto- $\alpha$-naphthol.}

Die Verseifung des Gattermannschen Äthyläthers mit Aluminiumchlorid erfolgt, wio bereits erwăhnt, weit besser in benzolischer als in Schwofelkohlenstofi-Lōsung. Das Benzol wird zweckmäßig vor der Verwendung durch Kochen mit 2-3\% Aluminjumehlorid von Thiophen. befreit. Für die Verseifung dient ein dem oben beschriebenen abnlicher Apparat. Auf $50 \mathrm{~g}$ des $\bar{A}$ thers wurden $200 \mathrm{ccm}$ Benzol und $75 \mathrm{~g}$ Aluminiumchlorid angewandt. Das Gemisch gerăt von selbst ins Sieden. Nach dem Abflauen der ersten Reaktion wurdo noch längere Zeit auf dem Wasserbade zum Sieden erhitzt, dann in verdünnte Salzsåure eingetragen, das Benzol mit Wasserdampf abgetrieben, der Rückstand in Äther aufgenommen und die ätherische Lösung in der sch'n angegebenen Weise durch Ausschütteln mit Sodalōsung und verdünter Natronlauge, jedes bis zur Erschöpfung, anfgearbejtet. Der vicht in Realtion getretene Acetonaphtholäther fand sich in dem ausgeschültelten ätherischen. Rückstand. Das aus der Sodalösung durch Salzsäure gefällte Acetonaphthol wurde durch Krystallisation aus Eisessig und T'oluol gereinigt und von dem Diacetonaphthol getrennt.

Farblose, ganz schwach gelbliche Prismen vom Schmp. 1980. Ausbeute $79 \%$ der'Theorie.

$01746 \mathrm{~g}$ Sbst.: $0.4945 \mathrm{~g} \mathrm{CO}, 0.0866 \mathrm{~g} \mathrm{H}_{\mathrm{g}} \mathrm{O} .-0.1632 \mathrm{~g}$ Sbst.: $0.4618 \mathrm{~g}$ $\mathrm{CO}_{2}, 00795 \mathrm{~g} \mathrm{H}_{2} \mathrm{O}$.

$$
\begin{array}{ll}
\mathrm{C}_{19} \mathrm{H}_{10} \mathrm{O}_{2} . & \text { Ber. C 77.42, } \mathrm{H} \text { 5.38. } \\
& \text { Gef. } ~ 77.24,77.17, \quad 5.55,5.45 .
\end{array}
$$

Das Oxim dieses Acetonaphthols wird durch Versetzen seiner verdiunten Lösung in Kalilauge oder noch besser nach der von Winterstein angegebenen ') Methode bergestellt und aus Toluol umkrystallisiert. Gut ausgebildete, lichtempfindliche, bei $164^{\circ}$ schmelzende Krystalle.

$0.1671 \mathrm{~g}$ Sbst.: $0.4372 \mathrm{~g} \mathrm{CO}, 0.0835 \mathrm{~g} \mathrm{H}_{2} \mathrm{O} .-0.1747 \mathrm{~g}$ Sbst.: $10.1 \mathrm{ccm}$ $\mathrm{N}\left(18.5^{\circ}, 768 \mathrm{~mm}\right)$.

$$
\begin{aligned}
& \mathrm{C}_{12} \mathrm{H}_{11} \mathrm{O}_{2} \mathrm{~N} \text {. Ber. C 71.64, H 5.47, N 6.97. } \\
& \text { Get. 》 71.36, 5.59, \$ } 6.84 .
\end{aligned}
$$

Versetzt man eine Lösung des $p$-Acetonaphthols in 50-prozentiger Essigsäure mit Phenylhydrazin, so bildet sich das bei $133^{\circ}$ schmelzende Phenylhydrazon. Auch die Oxydation des Acetonaphthols zu $\mathrm{Phthalsäure} \mathrm{mit} \mathrm{Hülfe} \mathrm{von} \mathrm{Kaliumpermanganat} \mathrm{wurde} \mathrm{in} \mathrm{verdünnter}$ essigsaurer Lösung vorgenommen.

1) B. 29, $1393[1896]$. 


\section{4-Diaceto- $\alpha-\mathrm{naph}$ thol.}

Dieses Diketon' wird in besonders reichlicher Menge neben dem $p$-Acetonaphthol erbalten, wenn man durch Erhöhung der Aluminiumchloridmenge die Energie des Verseifungsprozesses steigert. Bei dem Ausschüttelungsverfahren geht es mit dem Acetonaphthol in die Sodalösung über und wird daher mit ihm durch Salzsäure aus dieser Lösung gefällt. Bei der Krystallisation der Fällung aus Toluol findet es sich in den Mutterlaugen. Es wird durch Krystallisation aus Alkohol gereinigt. Feine, lange, farblose Nadeln vom Schmp. $140^{\circ}$.

$0.1767 \mathrm{~g}$ Sbst.: $0.4752 \mathrm{~g} \mathrm{CO}_{2}, 0.0838 \mathrm{~g} \mathrm{H}, \mathrm{O} .-0.1686 \mathrm{~g}$ Sbst.: $0.4405 \mathrm{~g}$ $\mathrm{CO}_{3}, 0.0760 \mathrm{~g} \mathrm{H}_{2} \mathrm{O}$.

$$
\begin{aligned}
& \mathrm{C}_{14} \mathrm{H}_{12} \mathrm{O}_{2} \text {. Ber. C 73.68, H 5.26. } \\
& \text { Gef. - 73.35, 73.43, } \text { 5.31, 5.20. }
\end{aligned}
$$

Versetzt man eine alkalische Lösung des Diacetonaphthols mit Hydroxylamin-chlorhydrat, so erhält man das schön krystallisierende, lichtempfindliche Monoxim vom Schmp. $212^{\circ}$, für welches wir den Eintritt der Oximidogruppe in den in der Stellung 2 befindlichen Acetylrest annehmen, da nach unserer Erfahrung das o-Acetonaphthol mit Hydroxylamin weit leichter reagiert, als die $p$-Verbindung.

$0.1733 \mathrm{~g}$ Sbst.: $0.4383 \mathrm{~g} \mathrm{CO}_{2}, 0.0838 \mathrm{~g} \mathrm{H}, 0 .-0.1801 \mathrm{~g}$ Sbst.: $8.6 \mathrm{ccm}$ $\mathrm{N}\left(17^{\circ}, 760.5 \mathrm{~mm}\right)$.

$$
\begin{aligned}
& \mathrm{C}_{14} \mathrm{H}_{18} \mathrm{O}_{3} \mathrm{~N} \text {. Ber. C 69.13, H 5.35, N 5.76. } \\
& \text { Gef. > 68.98, \5.41, } 5.62 \text {. }
\end{aligned}
$$

Behandelt man das Diacetonaphthol mit der kochenden Winterstein schen alkoholischen Hydroxylaminlösung, so erhält man das aus Toluol in feinen, farblosen, verfilzten, lichtempfindlichen Nädelchen vom Schmp. $220^{\circ}$ krystallisierende Dioxim.

$0.1685 \mathrm{~g}$ Sbst.: $0.4010 \mathrm{~g} \mathrm{CO}_{2}, 0.0835 \mathrm{~g} \mathrm{H} \mathrm{O} .-0.1715 \mathrm{~g}$ Sbst.: $15.5 \mathrm{ccm}$ N $\left(16^{\circ}, 762 \mathrm{~mm}\right)$.

$$
\begin{aligned}
& \mathrm{C}_{14} \mathrm{H}_{14} \mathrm{O}_{3} \mathrm{~N}_{2} . \\
& \text { Ber. C 65.12, H 5.43, N } 10.85 . \\
& \text { Gef. } 64.92, \times 5.54, * 10.70 .
\end{aligned}
$$

3-Aceto- $\beta$-naphthol-methyläther und 3-Aceto- $\beta$-naphthol.

Der von Gattermann bereits beschriebene Äther wurde nach dem Verfahren dieses Forschers hergestellt, wobei aber viel des angewandten $\beta$-Naphthol-methyläthers unverändert blieb, während von dem in Reaktion getretenen Anteil bis $\mathrm{zu} 61 \%$ der Theorie an dem gesuchten Keton erbalten wurden, der Rest aber sich in Form ron Verseifungsprodukten in der Soda-Ausschüttlung vorfand. Durch Steigerung der Mengen von Aluminiumchlorid konnte zwar eine gesteigerte Umsetzung des Rohmaterials erreicht werden, gleichzeitig aber nahmen die Neben- 
reaktionen überhand. Der aus der Ātherfraktion gewonnene 3-Aceto$\beta$-naphtbol-metbyläther wird am besten aus Ligroin umkrystallisiert und dabei in farblosen, vierseitigen Tafeln erbalten, welche bei $57.5-58^{\circ}$ schmelzen. Nach einem etwa gleichzeitig gebildeten Isomeren wurde vergeblich gefahndet.

Die Verseifung des Äthers wurde in der bereits beschriebenen Weise durchgeführt. Das gebildete Aceto- $\beta$-naphthol ist nur sebr schwach sauer, es geht daher nur schwierig in die Sodalösung über. Es ist leicht löslich in den meisten organischen Lösungsmitteln und wird am besten aus Petroleumäther umkrystallisiert und dabei in farblosen, blättrigen Krystallen rom Schmp. $64-65^{\circ}$ erbalten. Das Kaliumsalz biidet schöne, gelbe Blättchen. Ammoniakalische Lösungen des Ketons färben sich mit der Zeit schwarz.

$0.1827 \mathrm{~g}$ Sbst.: $0.5171 \mathrm{~g} \mathrm{CO}_{2}, 0.0883 \mathrm{~g} \mathrm{H}_{3} \mathrm{O} .-0.1707 \mathrm{~g}$ Sbst.: $0.4839 \mathrm{~g}$ $\mathrm{CO}_{2}, 0.0830 \mathrm{~g} \mathrm{H}_{2} \mathrm{O}$.

$$
\begin{aligned}
& \mathrm{C}_{12} \mathrm{H}_{10} \mathrm{O} \text {. Ber. C 77.42, H } 5.38 \text {. } \\
& \text { Gef. 》77.19, 77.31, > 5.41, } 54 t \text {. }
\end{aligned}
$$

Versetzt man eine Eisessiglösung dieses Acetonapthols tropfen weise mit etwas mehr als der berechneten Menge einer 90-prozentigen Lösung von Hydrazinhydrat, kocht dann auf und versetzt nach dem Abkühlen mit Wasser und etwas Salzäure, so scheidet sich das bereits besprochene $C$-Methyl- $\beta, \beta-n$ aphthindazol (S. 3225) aus, welches aus Alkohol in kleinen farblosen Nadeln rom Schmp. 217 krystallisiert und in heißer konzentrierter Kalilauge sowohl wie in konzentrierter Salzsäure löslich ist, ohne sich zu verändern. Das intermediär gebildete Hydrazon des Aceto- $\beta$-naphthols konnte nicht gefabt werden.

0.1792 g Sbst.: 0.5191 g $\mathrm{CO}_{2}, 0.0925 \mathrm{~g} \mathrm{H}_{2} \mathrm{O} .-0.1773 \mathrm{~g}$ Sbst.: $23.8 \mathrm{ccm}$ $\mathrm{N}\left(22^{\circ}, 755^{\circ} \mathrm{m} \iota \mathrm{m}\right)$.

$$
\begin{array}{ll}
\mathrm{C}_{12} \mathrm{H}_{10} \mathrm{~N}_{2} . & \text { Ber. C } 79.12, \mathrm{H} 5.49, \mathrm{~N} 15.38 . \\
& \text { Gef. } 779.00, \rtimes 5.77, \rtimes 15.38 .
\end{array}
$$

\section{6-Diaceto- $\beta$-naphthol.}

Dieser Körper entsteht, wie das isomere $\alpha$-Derivat als Nebenprodukt bei der Darstellung sowohl wie bei der Verseifung des Äthers. Es wird in derselben Weise isoliert, wie sein Isomeres und am besten aus Benzol umkrystallisiert, aus welchem es in kurzen Nädelchen vom Schmp. $165^{\circ}$ anschießt. In alkalischen Flüssigkeiten löst es sich mit gelber Farbe und das Kalium-uod Natriumsalz können in hübschen gelben Krystallen erhalten werden.

$0.1690 \mathrm{~g}$ Sbst.: $0.4547 \mathrm{~g} \mathrm{CO}_{2}, 0.0825 \mathrm{~g} \mathrm{H}_{2}$ O. $-0.1742 \mathrm{~g}$ Sbst.: $0.4707 \mathrm{~g}$ $\mathrm{CO}_{3}, 0.0863 \mathrm{~g} \mathrm{H}_{2} \mathrm{O}$.

$$
\begin{aligned}
& \mathrm{C}_{14} \mathrm{H}_{12} \mathrm{O}_{3} \text {. Ber. C 73.68, H } 5.26 \text {. } \\
& \text { Gef. } \gg 73.38,73.69, \times 5.46,5.54 \text {. }
\end{aligned}
$$


Mit überschlissigem Hydrazin längere Zeit am RückfluBkuhler gekocht, liefert dieses Diacetonaphthol das bereits erwähnte $\mathrm{Hydrazon}$ des Aceto-methyl-naphthindazols (S. 3226) in Form eines krystalliniscben gelben Niederscblags, welcber infolge seiner Unlöslicbkeit für die Analyse nur mit Eisessig, Alkobol und Ätber ausgewaschen wurde.

$0.1843 \mathrm{~g}$ Shst.: $0.2097 \mathrm{~g} \mathrm{CO}_{2}, 0.0911 \mathrm{~g} \mathrm{H}_{2} \mathrm{O},-0.1776 \mathrm{~g}$ Sbst.: $28.6 \mathrm{ccm}$ $\mathrm{N}(170,760.5 \mathrm{~mm})$.

$$
\begin{aligned}
\mathrm{C}_{28} \mathrm{H}_{24} \mathrm{~N}_{6} . & \text { Ber, C 75.68, HI 5.41, N } 18.92 . \\
& \text { Gef. } 75.42,-5.53 \text {, } 18.97 .
\end{aligned}
$$

Kocht man dieses Produkt mit konzentrierter Salzsäure, verdünnt mit Wasser und wāscht mit verdünnter Sodalösung und dann mit Wasser aus, so erbält man einen weißen Körper, welcher durch $K_{r y}$ stallisation aus Toluol und dann aus verdünnter Essigsäure in fächerförmig gruppierten Nadeln vom Schmp. $175^{\circ}$ erhalten wird. Er ist das

6 -Aceto- $C$-methyl$\beta, \beta$-napbthindazol,

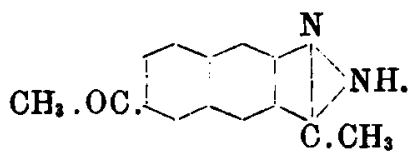

$0.1521 \mathrm{~g}$ Sbst.: $0.4172 \mathrm{~g} \mathrm{CO}_{2}, 0.0765 \mathrm{~g} \mathrm{H}_{2} \mathrm{O} .-0.1245 \mathrm{~g}$ Sbst.: $13.2 \mathrm{cem}$ $\mathrm{N}\left(17^{\circ}, 767 \mathrm{~mm}\right)$.

$$
\begin{aligned}
& \mathrm{C}_{14} \mathrm{H}_{12} \mathrm{ON}_{2} \text {. Ber. C 75.00, H 5.36, N } 12.50 \text {. } \\
& \text { Gef. > 71.81, 5.63, 》 } 12.59 \text {. }
\end{aligned}
$$

Dal3 das Diaceto- $\beta$-naphthol, ebenso wie das sogleich zu erwähnende 6-Aceto- $\beta$-vaphthol bei der Oxydation mit Kaliumpermanganat Trimellitsäure liefern, ist bereits hervorgeboben worden.

$$
6 \text { - Aceto- } \beta \text {-naphthol. }
$$

Dieses interessante weitere Isomere der Acetonaphthol-Reihe, welches ron allen Acetonaphtholen die stärkst sauren Eigenschaften besitzt, konnte nur mit sehr unbefriedigenden Ausbeuten durch Erbitzen von $\beta$-Naphthol-acetat mit Chlorzink und Eisessig auf Temperaturen von $150-160^{\circ}$ erbalten werden, wobei es uns trotz mebrfacher Änderungen der Reaktionsbedingungen nicht gelang, die Ausbeuten über etwa $5 \%$ der Theorie zu steigern. Weitaus die größte Menge des Reaktionsproduktes bestand regelmäßig aus teerigen Massen, welche nichts Faßbares lieferten. Die Isolierung des neuen Acetonaphthols gelang überbaupt nur durch Anwendung der SodaExtraktionsmethode. Es krystallisiert aus Benzol in kurzen sechsseitigen Prismen vom Schmp. $171^{\circ}$, löst sich wie alle Acetonaphthole in Alkalien mit gelber Farbe und gibt mit starken Laugen Ausscheidungen von gelben Krystallen seiner Alkalisalze. 
$0.1648 \mathrm{~g}$ Sbst.: $0.4660 \mathrm{~g} \mathrm{CO}, 0.0798 \mathrm{~g} \mathrm{H} \mathrm{H}_{2} \mathrm{O} .-0.1235 \mathrm{~g}$ Sbst.: $0.3511 \mathrm{~g}$ $\mathrm{CO}_{2}, 0.0617 \mathrm{~g} \mathrm{H}_{2} \mathrm{O}$.

$$
\begin{array}{ll}
\mathrm{C}_{12} \mathrm{H}_{10} \mathrm{O}_{2} . & \text { Ber. C 77.42, } \\
\text { Gef. }>77.12,77.53, & \text { H 5.38. } \\
\text { Ge.42, 5.59. }
\end{array}
$$

In eisessigsaurer Lösung mit Hydrazin versetzt, aufgekocht, mit Wasser und mit verdünntem Ammoniak gewaschen, liefert das neue Acetonaphthol das zugebörige Hydrazon, welches aus Pyridin umkrystallisiert und dabei in feinen gelben Nädelchen vom Schmp. 295 erhalten wird. Dieses Hydrazon ist in den meisten organischen Lösungsmitteln sehr schwer löslich, dagegen löst es sich leicht in verdünnten Alkalien. Durch Kochen mit einem Gemisch aus Eisessig und verdünnter Schwefelsäure zerfällt es wieder in die Substanzen, aus denen es entstanden war, in Hydrazin und Acetonaphthol.

$0.1596 \mathrm{~g}$ Sbst: $0.4570 \mathrm{~g} \mathrm{CO}_{3}, 0.0782 \mathrm{~g} \mathrm{H}_{2} 0 .-0.1782 \mathrm{~g}$ Sbst.: $11.3 \mathrm{ccm}$ $\mathrm{N}\left(17^{0}, 763 \mathrm{~mm}\right)$.

$$
\begin{array}{ll}
\mathrm{C}_{24} \mathrm{H}_{20} \mathrm{O}_{2} \mathrm{~N}_{2} . & \text { Ber. C 78.26, H } 5.43, \mathrm{~N} 7.61 \text {. } \\
\text { Gef. } 78.09 \text {, } ~ & 5.48, 》 749 .
\end{array}
$$

Berichtigungen.

Jahirg. 47, Heft 14, S. 2776, $160 \mathrm{~mm}$ v. 0. statt $>$ Sulfatesk lies "Sulfides 47 , > 16, S. 2981 , Spalte 1 nud 9 , ist $\rightarrow+\mathrm{H}_{2} \mathrm{O}_{2} \times$ zu strcichen. 